\title{
Improved Delivery of Caffeic Acid through Liposomal Encapsulation
}

\author{
Nuwanthi P. Katuwavila, ${ }^{1,2}$ A. D. L. Chandani Perera, ${ }^{1,3}$ V. Karunaratne, ${ }^{2,3}$ \\ Gehan A. J. Amaratunga, ${ }^{2,4}$ and D. Nedra Karunaratne ${ }^{1,3}$ \\ ${ }^{1}$ Postgraduate Institute of Science, University of Peradeniya, 20400 Peradeniya, Sri Lanka \\ ${ }^{2}$ Sri Lanka Institute of Nanotechnology, Mahenwatte, Pitipana, 10200 Homagama, Sri Lanka \\ ${ }^{3}$ Department of Chemistry, Faculty of Science, University of Peradeniya, 20400 Peradeniya, Sri Lanka \\ ${ }^{4}$ Electrical Engineering Division, Department of Engineering, University of Cambridge, $9 \mathrm{JJ}$ Thomson Avenue, \\ Cambridge CB3 OFA, UK
}

Correspondence should be addressed to Nuwanthi P. Katuwavila; nanukanp@yahoo.com

Received 10 November 2015; Revised 3 February 2016; Accepted 16 February 2016

Academic Editor: Yasuhiko Hayashi

Copyright (C) 2016 Nuwanthi P. Katuwavila et al. This is an open access article distributed under the Creative Commons Attribution License, which permits unrestricted use, distribution, and reproduction in any medium, provided the original work is properly cited.

Photoageing resulting from long term exposure of the skin to UV light can be minimized by scavenging the reactive photochemical intermediates with antioxidants. For effective photoprotection, the antioxidant must overcome the barrier properties of the skin and reach the target site in significant amounts. The present study aims to improve the skin penetration of caffeic acid, a very effective free radical scavenger, by encapsulating in liposomes. Caffeic acid loaded liposomes prepared using the reverse phase evaporation technique showed $70 \%$ encapsulation efficiency and size around $100 \mathrm{~nm}$ with zeta potential of $-55 \mathrm{mV}$. In vitro diffusion through a dialysis membrane enabled $70 \%$ release of encapsulated caffeic acid within $7 \mathrm{~h}$, whereas $95 \%$ of free caffeic acid diffused within $4 \mathrm{~h}$ in PBS solution ( $\mathrm{pH}$ 7.4). Liposomal caffeic acid permeation through pig skin epidermis in a Franz cell apparatus was $45 \%$ during $7 \mathrm{~h}$. In contrast, free caffeic acid was almost nonpermeable $(<5 \%)$ to pig skin during this time. The DPPH assay indicated that skin penetration did not destroy the antioxidant activity of liposomal caffeic acid or free caffeic acid. In conclusion, we confirm that liposomal caffeic acid may be successfully employed as an effective photoprotective agent against UV mediated skin damage.

\section{Introduction}

The exposure of skin to solar ultraviolet (UV) radiation causes skin damage resulting in both skin lesions and acceleration of skin ageing [1]. The UVB radiation preferentially acts on epidermis where it damages DNA in keratinocytes, while UVA is able to penetrate far deeper into the skin and hence exerts effects on both dermal and epidermal parts of the skin [2]. Further, the UV radiation causes the depletion of natural cellular antioxidants such as vitamins $\mathrm{A}, \mathrm{C}$, and $\mathrm{E}$, squalene, and coenzyme Q-10 resulting in the accumulation of reactive oxygen species (ROS): hydrogen peroxide, superoxide anion, singlet oxygen, hydroxyl radicals, and nitric oxide (NO) [3]. This imbalance between the production of ROS and reduction of antioxidant defences leads to cellular damage which disrupts the structural integrity of the skin thereby accelerating the ageing process [2]. The antioxidants react with and prevent formation of undesirable free radicals during the oxidation processes. Topical administration of antioxidants has been proved as a successful remedy for protecting the skin against UV mediated oxidative damage [4]. Caffeic acid (3,4-dihydroxycinnamic acid) is a hydroxycinnamic acid which is the major subgroup of phenolic compounds. These are secondary plant metabolites naturally produced in almost all plants as key intermediates in the biosynthesis of lignin. Caffeic acid can be isolated from the bark of Eucalyptus globulus Labill. [5]. In plants, it is biosynthesized from 4-hydroxycinnamic acid [6] and can be transformed into ferulic acid. These types of phenolics exhibit strong antioxidant activity. 
Caffeic acid is known to be an effective antioxidant in different in vitro antioxidant assays such as 1,1-diphenyl-2picryl-hydrazyl (DPPH) free radical scavenging, 2,2' -azinobis(3-ethylbenzothiazoline-6-sulphonic acid) (ABTS) radical scavenging, total antioxidant activity by ferric thiocyanate method, reducing power, and superoxide anion radical scavenging [7]. Caffeic acid and its derivatives can trap the free radicals directly or scavenge them through several coupled reactions [8]. Different approaches have been used to maximize the performance of these antioxidants because proper absorption into the skin and appropriate residence time at the target tissue are required to obtain the desired outcome [9].

However, the main obstacle to these topical applications is the blocking nature of the skin which prevents permeation of the reactive agent. The penetration of most drugs through the stratum corneum of the skin is more slow [10]. Thus, topically applied drugs are modified with a carrier system to allow penetration through the stratum corneum. Among various strategies followed to improve the efficacy of bioactive agents, liposomes figure importantly. Liposomes are composed of one or more lipid bilayers surrounding an equal number of aqueous compartments created from cholesterol and natural nontoxic lipids [11]. More importantly, liposomes are able to solubilize lipid molecules in their lipid bilayer as well as hydrophilic molecules in its aqueous core [12], such that the incorporated molecules receive protection within the liposomes [11], at the same time acting as drug reservoirs for slow drug release [13]. Though the liposomal mechanism of action is not fully understood, researches have proved enhanced topical delivery of liposomal bioactive agents. For example, a liposomal catechin formulation provided better efficiency of catechin delivery with limited skin disruption and good stability [14]. There are several studies based on ferulic acid (a hydroxycinnamic acid) loaded liposomes for improved efficacy. A study on the entrapment of ferulic acid in liposomes resulted in high solubility and improved biodistribution of ferulic acid [15].

In the work described, caffeic acid was incorporated in liposomes with a view of improving its topical delivery. Slow release, the influence of liposomes on the skin permeation, and the influence of liposomes on the antioxidant activity of caffeic acid were investigated.

\section{Materials and Methods}

2.1. Materials. Caffeic acid (98\%), cholesterol (99\%), egg phosphatidylcholine (99\%), sodium chloride, potassium chloride, potassium dihydrogen phosphate, and disodium hydrogen phosphate were purchased from Sigma-Aldrich Chemical Company (St. Louis, MO, USA). Methanol, chloroform, diethyl ether, ethanol, and acetone were of analytical grade. The adult pig ear was obtained from a local slaughter house. Snake skin dialysis tubing with a MW cut-off of 3500 was purchased from Thermo Scientific, USA.

Ethical clearance was granted by the Postgraduate Institute of Science, University of Peradeniya, Peradeniya, Sri Lanka.
2.2. Preparation of Caffeic Acid Loaded Liposomes. Caffeic acid encapsulated liposomes were prepared using the reverse phase evaporation method adapted from literature [16]. First, the lipid components, egg phosphatidylcholine $(2 \%, \mathrm{w} / \mathrm{v})$, cholesterol $(0.8 \%, \mathrm{w} / \mathrm{v})$, and caffeic acid $(0.02 \%, \mathrm{w} / \mathrm{v})$, were dissolved in $5.0 \mathrm{~mL}$ volume of a chloroform : methanol $(2: 1)$ mixture. Next, the solvent was evaporated using a rotary evaporator at $40^{\circ} \mathrm{C}$. Then, the lipid film was redissolved in the organic layer (diethyl ether). Then, $10.0 \mathrm{~mL}$ of $0.01 \mathrm{M}$ phosphate buffered saline (PBS) solution ( $\mathrm{pH} 7.4$ ) was added and the mixture was sonicated for 5-10 minutes in a bath type sonicator (Ultrasonic Bath, Grant). The organic solvent in the mixture was removed using a rotary evaporator, and another $5.0 \mathrm{~mL}$ of $0.01 \mathrm{M}$ PBS ( $\mathrm{pH} 7.4$ ) was added and allowed to equilibrate at room temperature. Finally, the nonencapsulated caffeic acid was separated by centrifugation immediately after liposome preparation at $16000 \mathrm{rpm}$ (Sigma 3-18, Laborzentrifugen, Germany) to obtain the caffeic acid loaded liposomes.

2.3. Determination of Encapsulation Efficiency. Encapsulation efficiency of caffeic acid encapsulated liposomes was determined using spectrophotometry. The absorbance of caffeic acid remaining in the supernatant after centrifugation was measured at $285 \mathrm{~nm}$ using the UV-Visible spectrophotometer (Shimadzu, UV-3600, UV-VIS-NIR, Japan). Then, the concentration was calculated from a calibration plot obtained for pure caffeic acid. Encapsulation efficiency was calculated as follows:

$$
\text { Encapsulation efficiency }=\frac{T_{c}-T_{s}}{T_{c}} \times 100,
$$

where $T_{c}$ is the total caffeic acid used and $T_{s}$ is the total caffeic acid present in the supernatant.

2.4. Determination of Particle Size and Zeta Potential of Caffeic Acid Loaded Liposomes. The average particle size and size polydispersity of the liposomes dispersed in PBS after centrifugation were determined by dynamic light scattering technique at $25^{\circ} \mathrm{C}$ using a particle size analyzer (Zetasizer Nano ZS, Malvern Instruments, UK) at a fixed scattering angle of $90^{\circ}$. The zeta potential of nanoparticles was measured using the zeta potential analyzer (Zetasizer Nano ZS, Malvern Instruments, UK). All measurements were performed in triplicate.

For time dependent changes the average particle size and zeta potential of the samples of liposomes stored in the dispersed forms at $4^{\circ} \mathrm{C}$ were measured after one month and two months.

2.5. Analysis of the Liposomal Morphology. Liposomal morphology was also examined by Scanning Electron Microscopy (SEM, SU6600, Hitachi, Japan) operating at 5 and $10 \mathrm{kV}$. A drop of the particle suspension was placed on a clean glass slide coated with poly-l-lysine and dried well. The dried specimen was subjected to gold sputtering and observed by SEM. 
2.6. In Vitro Release Study. The release characteristics of caffeic acid from liposomes were studied in PBS solution $(\mathrm{pH} 7.4)$ at $37^{\circ} \mathrm{C}$. $5 \mathrm{~mL}$ of caffeic acid loaded liposomes (135 ppm of caffeic acid) was trapped inside a snake skin dialysis membrane (MW cut-off 3500) and this was immersed in PBS $(25.0 \mathrm{~mL})$ at room temperature under mild agitation. Aliquots $(1.5 \mathrm{~mL})$ were withdrawn at predetermined time intervals and mixed well with $1.5 \mathrm{~mL}$ of $0.1 \mathrm{M}$ methanol. Next, the UV absorbance was recorded at $285 \mathrm{~nm}$ using the UVVisible spectrophotometer. The release medium was refreshed with $1.5 \mathrm{~mL}$ of medium after each withdrawal. All measurements were performed in triplicate. Using the calibration plot, the concentrations were calculated and hence the cumulative release percentages were determined.

Similarly, a diffusion study of free caffeic acid $(5 \mathrm{~mL}$ of $135 \mathrm{ppm}$ ) with a concentration similar to the encapsulated liposomal caffeic acid was carried out using the above procedure.

2.7. Ex Vivo Skin Permeation Study. The pig ear was separated from the cartilage using a scalpel and forceps and the fat layer was removed, without disturbing the hair follicles, to expose the epidermis. Then, a skin disk was punched and immersed in the acceptor medium (PBS pH 7.4) to equilibrate until used. Next, the skin disk was placed on a filter paper to remove the surface solution and fixed between donor and acceptor areas with the stratum corneum side up. The available surface area of the skin for permeation was $1.76 \mathrm{~cm}^{2}$. The donor compartment contained $2.5 \mathrm{~mL}$ of liposomal caffeic acid and the receiver compartment $10 \mathrm{~mL}$ of PBS ( $\mathrm{pH} \mathrm{7.4).}$ The temperature was maintained at $37^{\circ} \mathrm{C}$ by immersing the diffusion cell in a water bath. The receiver compartment was magnetically stirred at $300 \mathrm{rpm}$. Aliquots $(1.0 \mathrm{~mL})$ were removed from the receptor compartment at 1-hour intervals and refreshed with $1.0 \mathrm{~mL}$ of PBS solution. This was repeated during a period of 7 hours. After the experiment, the pig ear skin was removed from the setup and soaked in methanol well and checked for the skin deposited caffeic acid. Same procedure was repeated with a similar concentration of free caffeic acid as the liposomal caffeic acid. All experiments were done in triplicate.

Quantification of Caffeic Acid in Collected Aliquots. Fresh PBS $(1.0 \mathrm{~mL})$ and $0.1 \mathrm{M}$ methanol $(2.0 \mathrm{~mL})$ were added to each aliquot collected and mixed well. Then, the absorbance of each aliquot was measured at $285 \mathrm{~nm}$ using the UV-Visible spectrophotometer. The concentrations were calculated using a calibration plot and the cumulative skin permeation percentages were determined.

Calculation of Cumulative Drug Permeation (Q), Flux (J), and Permeation Coefficient $\left(K_{p}\right)$. The average steady state flux $(J)$ is given by the following equation:

$$
J=\frac{d Q}{d t} \frac{1}{A}
$$

where $A$ is surface area of the skin and $d Q / d t$ is the slope of the plot of $Q$ versus $t$.
The skin permeability coefficient $\left(K_{p}\right)$ is given by the equation

$$
K_{p}=\frac{\mathrm{J}}{\Delta C}
$$

where $\Delta C$ is drug concentration difference between the donor and the receptor at a given time.

The average permeability coefficient can be calculated using the equation

$$
\left(K_{p}\right) \text { ave }=\frac{\sum(J / \Delta C)}{N},
$$

where $N$ is number of intervals.

2.8. Determination of In Vitro Antioxidant Activity of Encapsulated Caffeic Acid. To determine the activity of liposomal caffeic acid after permeation through the dialysis membrane and pig ear skin, the following experiment was performed. Briefly, $2.5 \mathrm{~mL}$ from the release medium after $7 \mathrm{~h}$ of in vitro release experiment of caffeic acid loaded liposomes through dialysis membrane and pig ear skin was added separately to $0.6 \mathrm{mM}$ DPPH in methanol $(0.5 \mathrm{~mL})$ and topped up to $5.0 \mathrm{~mL}$ with methanol. This reaction mixture was kept in the dark for 30 minutes and then the absorbance was measured at $514 \mathrm{~nm}$ using the UV-Visible spectrophotometer. $\mathrm{DPPH}$ in methanol was used as the control. DPPH assay was performed according to the procedure described by Braca et al. [17]. The antioxidant activity of a standard solution of caffeic acid of similar concentration of caffeic acid obtained from the release media after $7 \mathrm{~h}$ of permeation experiments through dialysis membrane and pig ear skin, respectively, was measured and compared with the antioxidant activity of permeated caffeic acid in both experiments. The DPPH radical scavenging activity was calculated using the following formula:

$$
\begin{aligned}
& \text { DPPH radical scavenging activity (\%) } \\
& \quad=\left(\frac{A_{c}-A_{s}}{A_{c}}\right) \times 100,
\end{aligned}
$$

where $A_{c}$ is the absorbance of the control and $A_{s}$ is the absorbance of the sample.

\section{Results and Discussion}

3.1. Characterization of Caffeic Acid Loaded Liposomes. The macroscopic appearance of the liposomal suspension was milky with a slight yellowish reflection characteristic of nanometric suspensions [18]. Caffeic acid loaded liposomes were characterized for their size, zeta potential, encapsulation efficiency, and morphology. The size, zeta potential, and encapsulation efficiency of nanoparticles were tabulated in Table 1. The average size of unloaded liposomes obtained from the dynamic light scattering technique was around $100 \mathrm{~nm}$ and the size distribution graph showed a Gaussian distribution of sizes of around 80-120 nm. This observation strongly supports the claim that liposomes within the size 
TABLE 1: The size, zeta potential, and \% EE of unloaded liposomes and caffeic acid loaded liposomes (each value represents the mean \pm SD, $n=3)$.

\begin{tabular}{lccc}
\hline System & Average size/nm & Zeta potential/mV & \% encapsulation efficiency \\
\hline Unloaded liposomes & $100 \pm 27$ & $-40 \pm 3$ & - \\
Caffeic acid loaded liposomes & $100 \pm 35$ & $-55 \pm 4$ & $70 \pm 4$ \\
\hline
\end{tabular}

TABLE 2: Time dependent changes in the size and zeta potential of caffeic acid loaded liposomes and unloaded liposomes (each value represents the mean $\pm \mathrm{SD}, n=3$ ).

\begin{tabular}{|c|c|c|c|c|c|c|}
\hline \multirow{2}{*}{ Systems } & \multicolumn{3}{|c|}{ Average size/nm } & \multicolumn{3}{|c|}{ Zeta potential/mV } \\
\hline & 0 months & 1 month & 2 months & 0 months & 1 month & 2 months \\
\hline Unloaded liposomes & $100 \pm 27$ & $112 \pm 23$ & $110 \pm 31$ & $-40 \pm 3$ & $-42 \pm 5$ & $-44 \pm 7$ \\
\hline Caffeic acid loaded liposomes & $100 \pm 35$ & $104 \pm 34$ & $108 \pm 28$ & $-55 \pm 4$ & $-52 \pm 6$ & $-49 \pm 4$ \\
\hline
\end{tabular}

range of 25-100 $\mathrm{nm}$ are small unilamellar type vesicles [19]. According to du Plessis et al., the intermediate sized liposomes $(100-200 \mathrm{~nm})$ show a better skin penetration suggesting the size dependence of liposomes in topical delivery [20]. So the liposomes we obtained might have a favourable effect on skin penetration.

The high negative zeta potential of the loaded liposomes $(-55 \mathrm{mV})$ is attributed to caffeic acid being a negatively charged molecule. Zeta potential is a good index of the magnitude of repulsive interactions between colloidal particles and it is commonly used to assess the stability of a nanosuspension. A high positive or negative value for zeta potential indicates that the repulsion between particles is high; hence, the colloidal dispersion would show good stability.

The liposomes obtained from reverse phase evaporation technique showed relatively high encapsulation efficiency of $70 \%$. The reverse phase evaporation technique is more suitable since the encapsulant, caffeic acid, is poorly water soluble and also this technique results in high encapsulation efficiencies [16]. According to Caddeo et al., generally, liposomal encapsulation was not significantly influenced by the lipid composition [21]. This high encapsulation efficiency is related to the poor water solubility of caffeic acid which might incorporate entirely with lipid bilayer by this technique.

In the stability testing, a significant difference in the average sizes as well as in the zeta potentials of unloaded liposomes and caffeic acid liposomes could not be observed (Table 2). After one month, the average size and the zeta potential of unloaded liposomes were $112 \pm 23 \mathrm{~nm}$ and $-42 \pm 5 \mathrm{mV}$, respectively, whereas with the caffeic acid loaded liposomes these were $104 \pm 34 \mathrm{~nm}$ and $-52 \pm 4 \mathrm{mV}$, respectively. After two months' time period also not any noticeable difference in average sizes of both systems was observed (Table 2). Though a slight drop with the zeta potential of caffeic acid loaded liposomes $(-49 \pm 4 \mathrm{mV})$ was observed, still the parameters indicate a stable colloidal suspension. This is in accordance with a previously published study on sodium ascorbyl phosphate loaded liposomes [22].

Morphological analysis of these liposomes by SEM showed spherical shaped 3D globular vesicles (Figure 1).

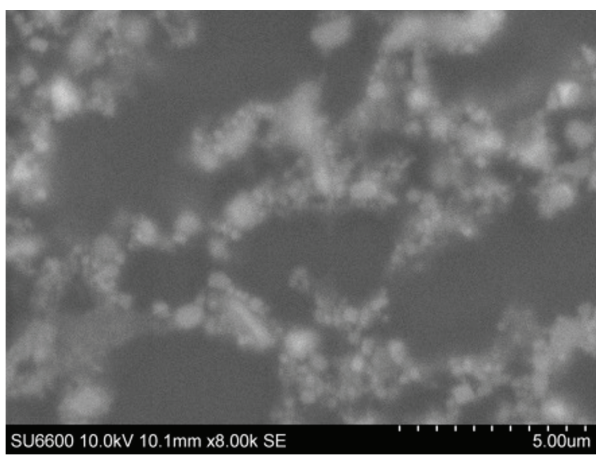

FIGURE 1: SEM image of caffeic acid loaded liposomes.

3.2. In Vitro Release Studies of Caffeic Acid Loaded Liposomes. In order to obtain qualitative and quantitative information, the release properties of liposomal caffeic acid and free caffeic acid were determined in PBS solution ( $\mathrm{pH}$ 7.4). In this study using the dialysis membrane method, kinetics showed an initial burst release of liposomal caffeic acid of $45 \%$ within $2 \mathrm{~h}$ of diffusion which was considered important for attaining higher levels of caffeic acid for skin penetration (Figure 2(b)). A continuous increase in the cumulative release up to $71 \%$ could be observed in the following 2 to $6 \mathrm{~h}$. After $24 \mathrm{~h}$, only a $1 \%$ increment in the cumulative release amount was attained. The remaining 30\% of liposomal caffeic acid might be permanently trapped inside the liposomes. When release of liposomal caffeic acid in the experiment above is compared to the release of free caffeic acid, a clear difference could be observed. Free caffeic acid diffuses rapidly where almost $50 \%$ had diffused after $2 \mathrm{~h}$. Within $4 \mathrm{~h}, 95 \%$ of the free caffeic acid permeated through the dialysis membrane (Figure 2(a)), indicating that liposomal encapsulation enables slow release up to $7 \mathrm{~h}$. This observation is further supported by the study of Fočo et al., who investigated liposomes as carriers of sodium ascorbyl phosphate for cutaneous photoprotection. Their in vitro diffusion study was performed in PBS with cellulose membrane and they reported $80 \%$ of liposomal release within $8 \mathrm{~h} \mathrm{[22].}$ 
TABLE 3: Permeability results obtained for free caffeic acid and liposomal caffeic acid for the permeation through pig ear epidermis.

\begin{tabular}{lcccc}
\hline Formulation & Study period $(\mathrm{h})$ & Percent skin permeation $(\%)$ & Percent skin deposition $(\%)$ & $K_{p}{\text { average }\left(10^{-3} \mathrm{~cm} \mathrm{~min}^{-1}\right)}$ \\
\hline Free caffeic acid & 7 & $5.3 \pm 1.91$ & $6.38 \pm 3.10$ & 1.33 \\
Liposomal caffeic acid & 7 & $41.8 \pm 1.58$ & $2.0 \pm 0.02$ & 28.19 \\
\hline
\end{tabular}

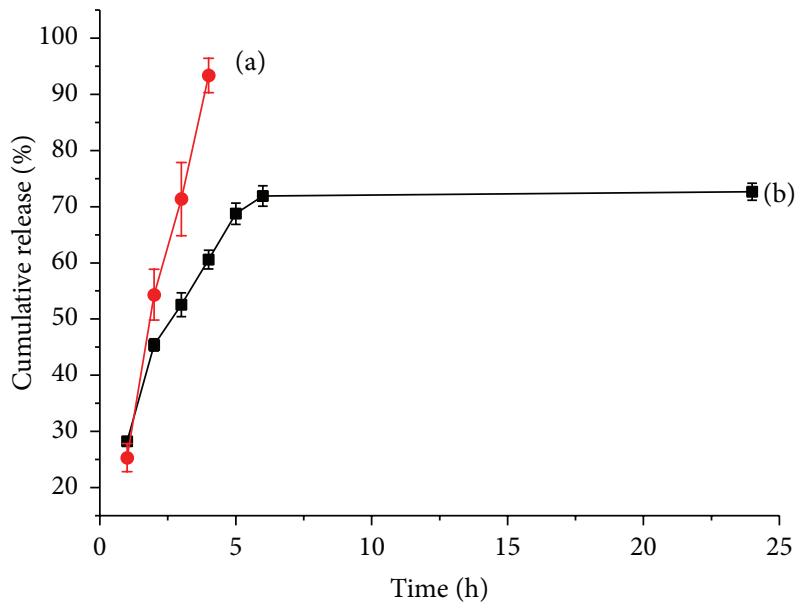

FIGURE 2: In vitro cumulative release profiles of (a) free caffeic acid and (b) liposomal caffeic acid through dialysis membrane at predetermined time intervals in $\mathrm{pH}$ 7.4 PBS. Data points represent mean cumulative release $( \pm \mathrm{SD})$ calculated from 3 samples.

3.3. Ex Vivo Skin Permeability Study. Considering the slow release of caffeic acid from liposomes, the effect of liposomal caffeic acid on skin permeation was studied. The percutaneous penetration of liposomal caffeic acid and free caffeic acid was compared using a Franz cell. The aim of this study was to determine whether the encapsulated caffeic acid has an improvement in permeation through the epidermis. According to the permeation profile for liposomal caffeic acid (Figure 3(a)), a considerable amount of caffeic acid (41.8 \pm $1.58 \%$ ) had permeated slowly through the epidermis during $7 \mathrm{~h}$ while only $2.0 \pm 0.02 \%$ of caffeic acid had deposited in the skin. The standard errors among some cumulative skin permeation values $(2-5 \mathrm{~h})$ were higher probably due to the differences among skin donors in the triplicate study. Free caffeic acid penetration was $5.3 \pm 1.91 \%$ (Figure $3(\mathrm{~b})$ ), which is significantly lower than liposomal caffeic acid, whereas the skin deposition of free caffeic acid was $6.38 \pm 3.10 \%$ which is slightly higher than the deposition of liposomal caffeic acid. Liposomal caffeic acid permeation was almost eight times higher than the amount of free caffeic acid penetrated through the epidermis (Table 3). This is further corroborated by the values obtained for the average permeation coefficient ( $K_{p}$ average) in Table 3 . The highest average permeability $\left(K_{p}\right.$ average $\left.=28.19 \times 10^{-3} \mathrm{~cm} \mathrm{~min}^{-1}\right)$ for pig ear was obtained with liposomal caffeic acid whereas the average permeation coefficient for free caffeic acid was $1.33 \times 10^{-3} \mathrm{~cm} \mathrm{~min}^{-1}$ indicating poor skin permeation of free caffeic acid through pig ear epidermis.

The interaction between skin and liposomes themselves may be an important contributory factor for this increase in

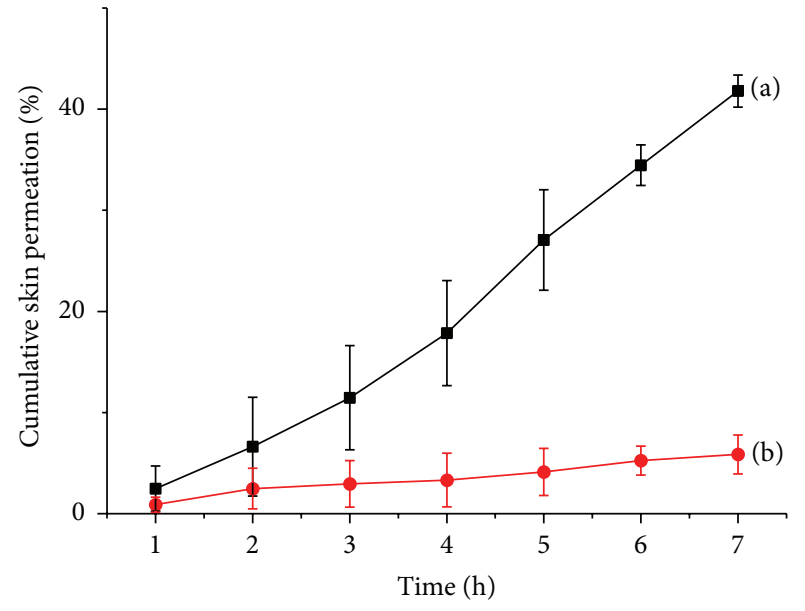

FIGURE 3: Ex vivo penetration profiles of (a) liposomal caffeic acid and (b) free caffeic acid through pig ear epidermis at predetermined time intervals in $\mathrm{pH}$ 7.4 PBS. Data points represent mean cumulative release $( \pm S D)$ calculated from 3 samples.

penetration of caffeic acid. Phosphatidylcholine may serve as a skin penetration enhancer interacting with and hydrating the stratum corneum, facilitating the penetration of liposomal encapsulants [23]. Free caffeic acid which showed 95\% diffusion through the dialysis membrane could only show $5 \%$ penetration of the pig skin. This might be due to the lower barrier effect of the dialysis membrane compared to the complex pig ear skin. The dialysis membrane is composed of cellulose while the skin is a complex lipid bilayer membrane. The cumulative liposomal caffeic acid percentage permeated through pig skin warrants further studies. For example, the skin penetration of liposomal sodium ascorbyl phosphate was around $50 \%$ after $8 \mathrm{~h}$ [22] which compares well with our study. In addition, several studies have confirmed that the amount of penetrated compound was significantly higher from liposomal formulations than from aqueous solution [3]. Therefore, encapsulation in liposomes would improve topical delivery of caffeic acid through the skin.

3.4. In Vitro Antioxidant Activity. The in vitro antioxidant activity of liposomal caffeic acid after the dialysis membrane and skin permeation experiments was determined by the DPPH free radical scavenging assay. From this method it is possible to determine the antioxidant power of an organic compound by measuring the decrease in absorbance of DPPH radical. The dialysis membrane permeated liposomal caffeic acid after $7 \mathrm{~h}$ showed $83.36 \pm 1.62 \%$ of free radical scavenging activity whereas standard caffeic acid at the same concentration was $86.18 \pm 1.50 \%$ (Figure 4). Similarly, the antioxidant activity of liposomal caffeic acid permeated after 


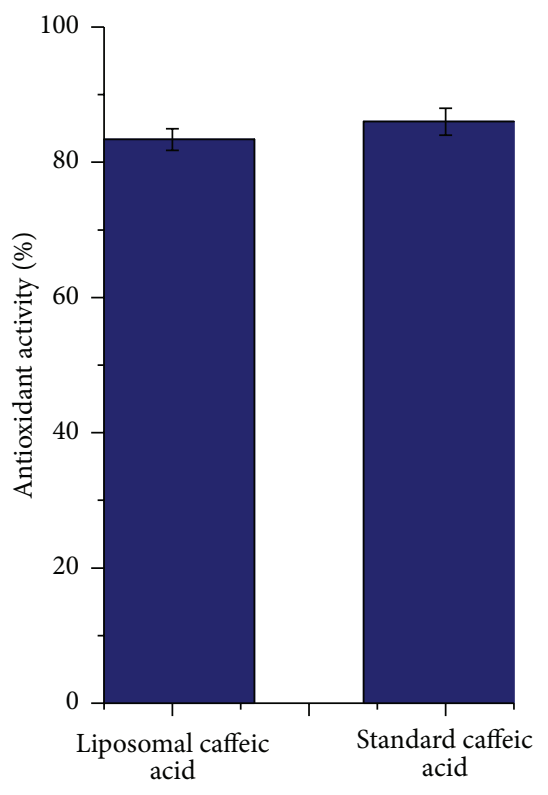

FIGURE 4: A comparison of percent antioxidant activity of liposomal caffeic acid after membrane permeation with that of the standard caffeic acid. Results are represented as the mean \pm SD of three experiments.

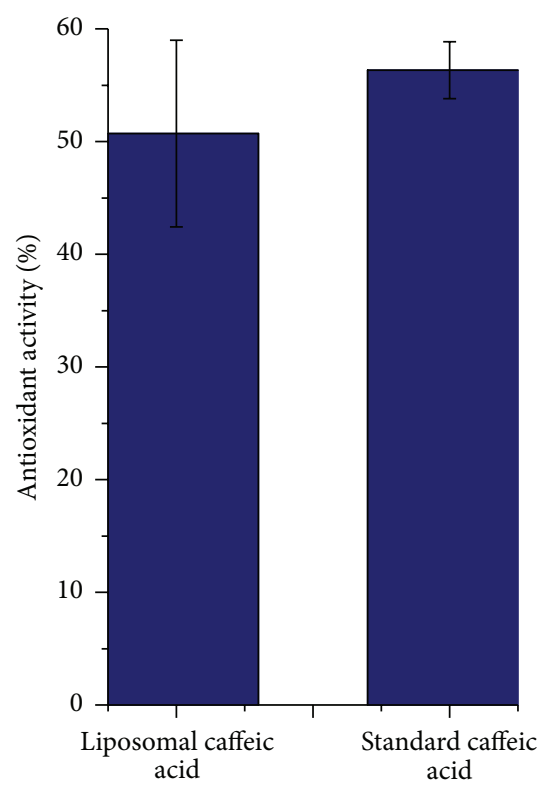

FIGURE 5: A comparison of percent antioxidant activity of liposomal caffeic acid after pig ear epidermis permeation with that of the standard caffeic acid. Results are represented as the mean \pm SD of three experiments.

$7 \mathrm{~h}$ of skin permeation experiment was $51.07 \pm 11.68 \%$ while the caffeic acid standard of same concentration had an activity of $56.66 \pm 3.21 \%$ (Figure 5). Clearly, encapsulation with liposomes does not destroy the antioxidant activity of caffeic acid permeating through the two types of membranes.
These values further reflect the amount of caffeic acid penetrating through the dialysis membrane (71\%) and the pig skin $(41.8 \%)$.

\section{Conclusion}

Caffeic acid loaded liposomes with high encapsulation efficiency $(70 \%)$ and of average size of $100 \mathrm{~nm}$ and high negative zeta potential $-55 \mathrm{mV}$ were prepared. These liposomes were able to achieve a cumulative release of $71 \%$ of entrapped caffeic acid within $7 \mathrm{~h}$ in PBS solution ( $\mathrm{pH} 7.4)$. The results of the pig ear epidermal membrane experiment proved the enhanced penetration of liposomal caffeic acid in comparison to free caffeic acid applied to the skin surface, suggesting it as a potential delivery system for the antioxidant caffeic acid. There was neither a reduction nor a synergic effect on the antioxidant activity of caffeic acid after encapsulation in liposomes, but the original activity was retained. The data from this study support the claim that liposomal encapsulation could improve the penetration of caffeic acid through the epidermis which could reduce the photodamage to the skin. Further, this formulation has the feasibility of developing as a skin cream formulation to successfully slow down the complex process of photooxidative skin damage.

\section{Conflict of Interests}

The authors declare that there is no conflict of interests regarding the publication of this paper.

\section{Acknowledgment}

This research was financially supported by the HETC QIG Win 3 grant, University of Peradeniya, Peradeniya, Sri Lanka.

\section{References}

[1] F. Gruber, V. Peharda, M. Kaštelan, and I. Brajac, "Occupational skin diseases caused by UV radiation," Acta Dermatovenerologica Croatica, vol. 15, no. 3, pp. 191-198, 2007.

[2] M. Ichihashi, "Photoaging of the skin," Japanese Society of AntiAging Medicine, vol. 6, no. 6, pp. 46-59, 2009.

[3] M. Podda, M. G. Traber, C. Weber, L.-J. Yan, and L. Packer, "UVirradiation depletes antioxidants and causes oxidative damage in a model of human skin," Free Radical Biology \& Medicine, vol. 24, no. 1, pp. 55-65, 1998.

[4] C. Weber, M. Podda, M. Rallis, J. J. Thiele, M. G. Traber, and L. Packer, "Efficacy of topically applied tocopherols and tocotrienols in protection of murine skin from oxidative damage induced by UV-irradiation," Free Radical Biology \& Medicine, vol. 22, no. 5, pp. 761-769, 1997.

[5] S. A. O. Santos, C. S. R. Freire, M. R. M. Domingues, A. J. D. Silvestre, and C. P. Neto, "Characterization of phenolic components in polar extracts of eucalyptus globulus labill. Bark by high-performance liquid chromatography-mass spectrometry," Journal of Agricultural and Food Chemistry, vol. 59, no. 17, pp. 9386-9393, 2011.

[6] B. M. R. Bandara, C. M. Hewage, V. Karunaratne, and N. K. B. Adikaram, "Antifungal principle of the rhizome of Costusspecious," Planta Medica, vol. 54, no. 5, pp. 477-478, 1988. 
[7] I. Gülçin, "Antioxidant activity of caffeic acid (3,4-dihydroxycinnamic acid)," Toxicology, vol. 217, no. 2-3, pp. 213-220, 2006.

[8] M. Rao, G. Paliyath, and D. Ormrod, "Ultraviolet-B- and ozone-induced biochemical changes in antioxidant enzymes of Arabidopsis thaliana," Plant Physiology, vol. 110, no. 1, pp. 125136, 1996.

[9] H. Sies, "Oxidative stress: oxidants and antioxidants," Experimental Physiology, vol. 82, no. 2, pp. 291-295, 1997.

[10] D. I. J. Morrow, P. A. McCarron, A. D. Woolfson, and R. F. Donnelly, "Innovative strategies for enhancing topical and transdermal drug delivery," The Open Drug Delivery Journal, vol. 1, pp. 36-59, 2007.

[11] K. Shashi, K. Satinder, and P. Bharat, "A complete review on: liposomes," International Research Journal of Pharmacy, vol. 3, no. 7, pp. 10-17, 2012.

[12] A. K. Sailaja and P. Shashikala, "An overall review on liposomal drug delivery systems," Indian Journal of Novel Drug Delivery, vol. 6, no. 2, pp. 112-119, 2014.

[13] G. M. M. El Maghraby, A. C. Williams, and B. W. Barry, "Can drug-bearing liposomes penetrate intact skin?" Journal of Pharmacy and Pharmacology, vol. 58, no. 4, pp. 415-429, 2006.

[14] J.-Y. Fang, T.-L. Hwang, Y.-L. Huang, and C.-L. Fang, "Enhancement of the transdermal delivery of catechins by liposomes incorporating anionic surfactants and ethanol," International Journal of Pharmaceutics, vol. 310, no. 1-2, pp. 131-138, 2006.

[15] J. Qin, D. Chen, W. Lu et al., "Preparation, characterization, and evaluation of liposomal ferulic acid in vitro and in vivo," Drug Development and Industrial Pharmacy, vol. 34, no. 6, pp. 602608, 2008.

[16] M. Riaz, "Liposomes preparation method," Pakistan Journal of Pharmaceutical Sciences, vol. 19, no. 1, pp. 65-77, 1996.

[17] A. Braca, N. De Tommasi, L. Di Bari, C. Pizza, M. Politi, and I. Morelli, "Antioxidant principles from Bauhinia tarapotensis," Journal of Natural Products, vol. 64, no. 7, pp. 892-895, 2001.

[18] M. C. B. Lira, M. S. Ferraz, D. G. V. C. da Silva et al., "Inclusion complex of usnic acid with $\beta$-cyclodextrin: characterization and nanoencapsulation into liposomes," Journal of Inclusion Phenomena and Macrocyclic Chemistry, vol. 64, no. 3-4, pp. 215224, 2009.

[19] F. M. Cagdas, N. Ertugral, S. Bucak, and N. Z. Atay, "Effect of preparation method and cholesterol on drug encapsulation studies by phospholipid liposomes," Pharmaceutical Development and Technology, vol. 16, no. 4, pp. 408-414, 2011.

[20] J. du Plessis, C. Ramachandran, N. Weiner, and D. G. Müller, "The influence of particle size of liposomes on the deposition of drug into skin," International Journal of Pharmaceutics, vol. 103, no. 3, pp. 277-282, 1994.

[21] C. Caddeo, K. Teskač, C. Sinico, and J. Kristl, "Effect of resveratrol incorporated in liposomes on proliferation and UVB protection of cells," International Journal of Pharmaceutics, vol. 363, no. 1-2, pp. 183-191, 2008.

[22] A. Fočo, M. Gašperlin, and J. Kristl, "Investigation of liposomes as carriers of sodium ascorbyl phosphate for cutaneous photoprotection," International Journal of Pharmaceutics, vol. 291, no. 1-2, pp. 21-29, 2005.

[23] M. Kirjavainen, A. Urtti, I. Jääskeläinen et al., "Interaction of liposomes with human skin in vitro-the influence of lipid composition and structure," Biochimica et Biophysica Acta, vol. 1304, no. 3, pp. 179-189, 1997. 

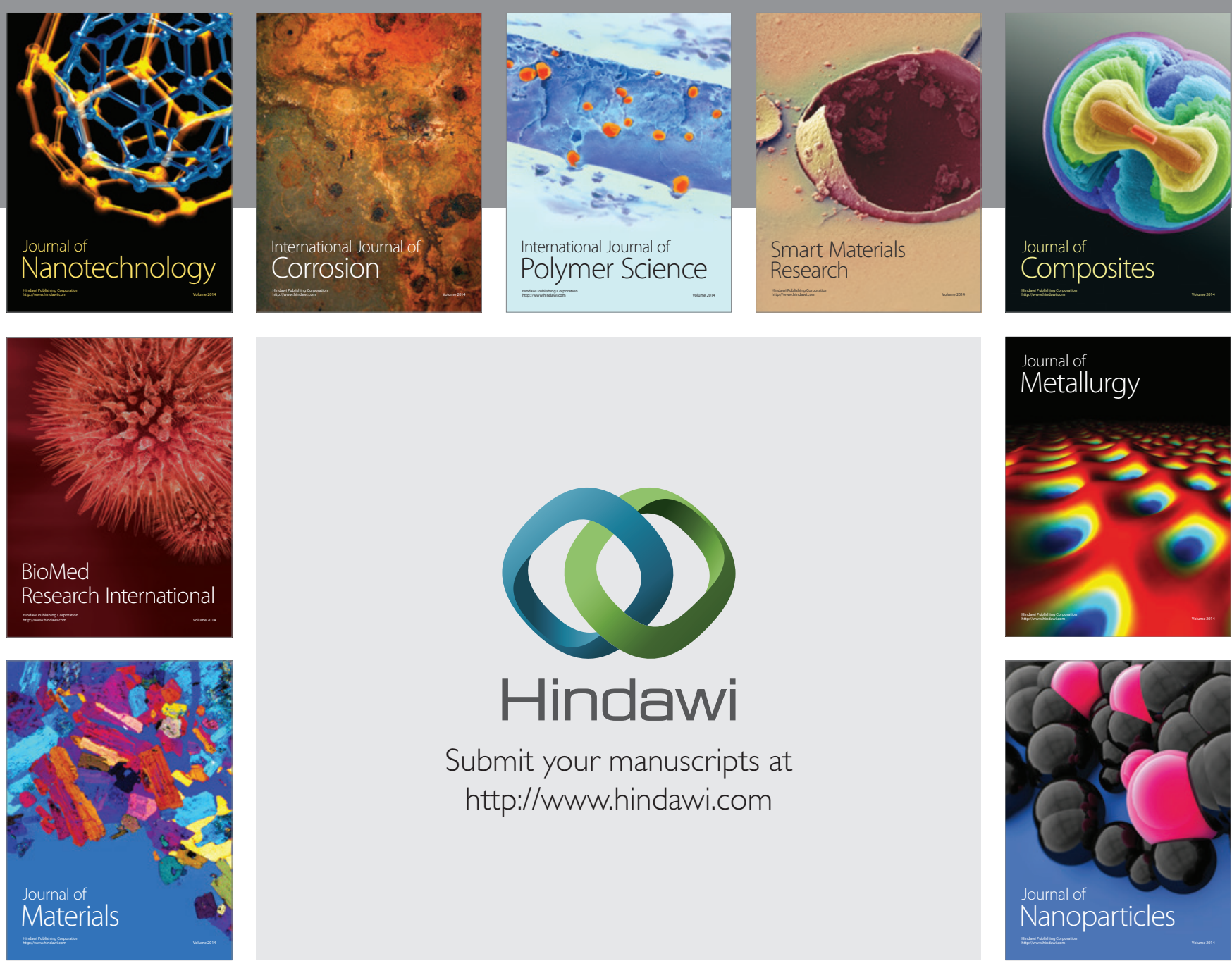

\section{Hindawi}

Submit your manuscripts at

http://www.hindawi.com

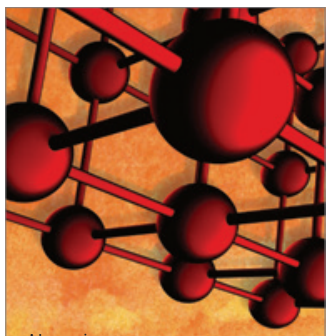

Materials Science and Engineering
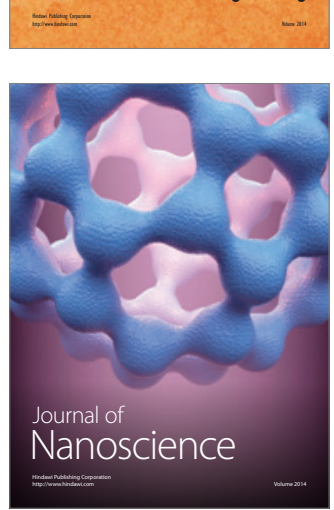
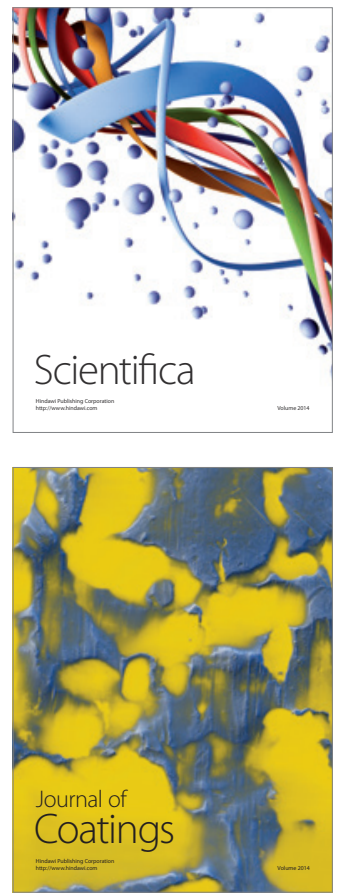
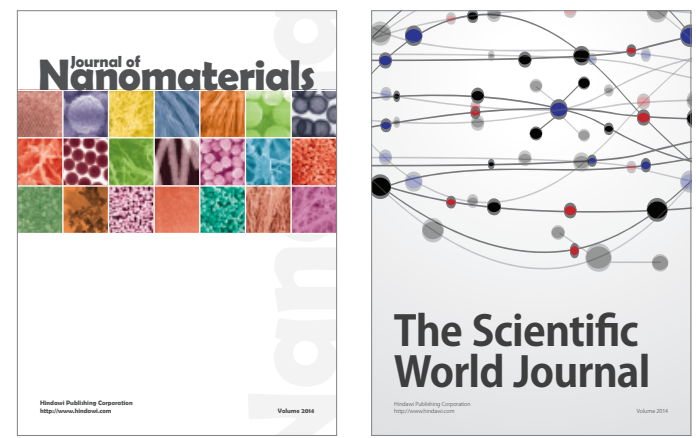

The Scientific World Journal
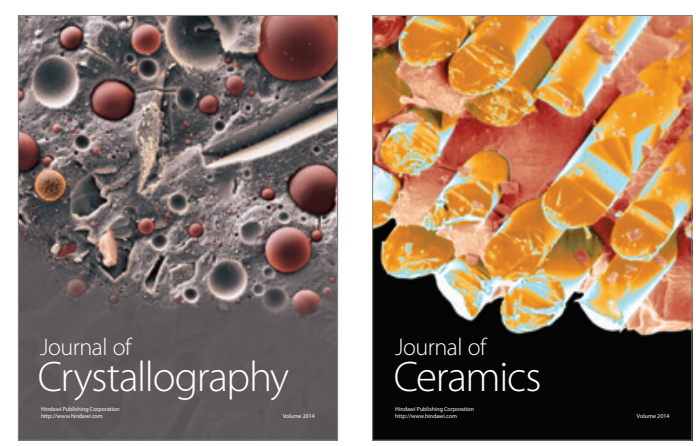
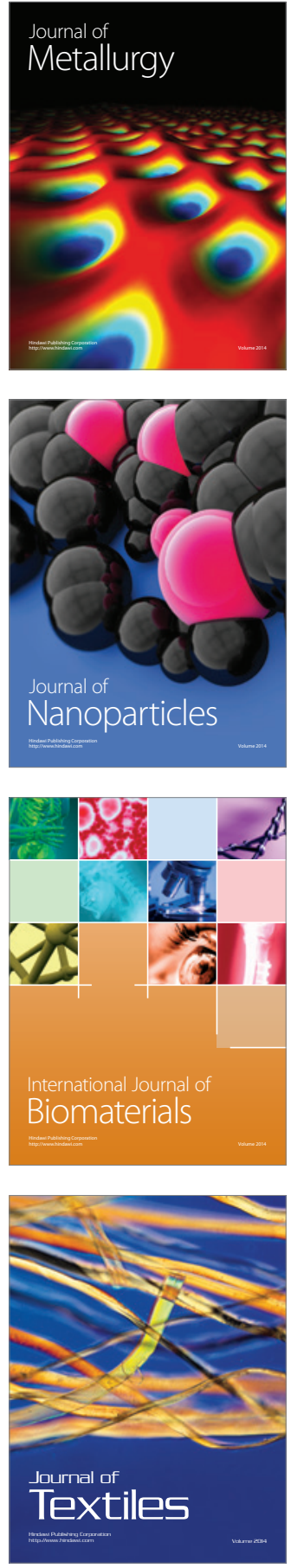